\title{
Ocular-specific ER stress reduction rescues glaucoma in murine glucocorticoid-induced glaucoma
}

\author{
Gulab S. Zode, ${ }^{1}$ Arti B. Sharma, ${ }^{1}$ Xiaolei Lin, ${ }^{1}$ Charles C. Searby, ${ }^{1,2}$ Kevin Bugge,, ${ }^{1,2}$ \\ Gun Hee Kim, ${ }^{1}$ Abbot F. Clark, ${ }^{3}$ and Val C. Sheffield ${ }^{1,2}$ \\ ${ }^{1}$ Department of Pediatrics and ${ }^{2}$ Howard Hughes Medical Institute, University of lowa, lowa City, Iowa, USA. \\ ${ }^{3}$ Department of Cell Biology and Immunology and North Texas Eye Research Institute, \\ University of North Texas Health Science Center at Fort Worth, Fort Worth, Texas, USA.
}

\begin{abstract}
Administration of glucocorticoids induces ocular hypertension in some patients. If untreated, these patients can develop a secondary glaucoma that resembles primary open-angle glaucoma (POAG). The underlying pathology of glucocorticoid-induced glaucoma is not fully understood, due in part to lack of an appropriate animal model. Here, we developed a murine model of glucocorticoid-induced glaucoma that exhibits glaucoma features that are observed in patients. Treatment of WT mice with topical ocular $0.1 \%$ dexamethasone led to elevation of intraocular pressure (IOP), functional and structural loss of retinal ganglion cells, and axonal degeneration, resembling glucocorticoid-induced glaucoma in human patients. Furthermore, dexamethasoneinduced ocular hypertension was associated with chronic ER stress of the trabecular meshwork (TM). Similar to patients, withdrawal of dexamethasone treatment reduced elevated IOP and ER stress in this animal model. Dexamethasone induced the transcriptional factor CHOP, a marker for chronic ER stress, in the anterior segment tissues, and Chop deletion reduced ER stress in these tissues and prevented dexamethasone-induced ocular hypertension. Furthermore, reduction of ER stress in the TM with sodium 4-phenylbutyrate prevented dexamethasone-induced ocular hypertension in WT mice. Our data indicate that ER stress contributes to glucocorticoid-induced ocular hypertension and suggest that reducing ER stress has potential as a therapeutic strategy for treating glucocorticoid-induced glaucoma.
\end{abstract}

\section{Introduction}

Glaucoma is the second leading cause of visual impairment and blindness worldwide, affecting about 70 million people, and is the leading cause of blindness among African-Americans in the United States (1-3). Primary open-angle glaucoma (POAG) is the most common form of glaucoma, accounting for approximately $70 \%$ of all cases $(2,4,5)$. POAG is characterized by progressive loss of retinal ganglion cell (RGC) axons and irreversible loss of vision $(2,4)$. Although the exact mechanisms that cause glaucoma are poorly understood, elevated intraocular pressure (IOP) is a major associated risk factor and a major target of treatment $(6,7)$. In glaucoma, the resistance to aqueous humor outflow through the drainage structure of the trabecular meshwork (TM) is increased, thus elevating $\operatorname{IOP}(4,8,9)$. The mechanisms that cause increased outflow resistance in the TM are not well understood.

Glucocorticoid therapy can elevate IOP in many susceptible individuals. Approximately $30 \%-40 \%$ of the general population can develop IOP elevation after topical ocular administration of a potent glucocorticoid (such as dexamethasone or betamethasone) for 4-6 weeks (10-16). If undetected, glucocorticoid-induced ocular hypertension can lead to secondary open-angle glaucoma that mimics POAG (14). Similar to POAG, glucocorticoid-induced ocular hypertension is caused by increased aqueous humor outflow resistance in the $\operatorname{TM}(11,15,17,18)$. However, the molecular mechanisms that lead to this increased resistance are still unknown.

Conflict of interest: Gulab S. Zode's research is partially funded by Alcon Laboratories Inc.

Citation for this article: J Clin Invest. 2014;124(5):1956-1965. doi:10.1172/JCI69774.
The ER is involved in the synthesis and processing of secreted and membrane proteins. Properly folded proteins are transported to the Golgi network, whereas misfolded proteins are retained in the ER and are removed by ER-associated degradation (ERAD). When the synthesis of secretory proteins exceeds the capacity of ER chaperones and the ERAD machinery, these proteins accumulate in the ER. Unfolded proteins tend to aggregate, leading to ER stress (19-21). To alleviate such cellular stress, eukaryotic cells activate a cytoprotective response, known as the ER stress response or the unfolded protein response (UPR). Activation of the UPR involves sensing of ER stress via PERK, ATF- $6 \alpha$, and IRE1, which then regulate the response to ER stress by inducing ER chaperones (GRP78 and GRP94), phosphorylation of eIF2 $\alpha$, activation of ATF-4, and alternative splicing of XBP-1 $(19,22)$. When the UPR adaptive response is not sufficient to resolve defects in protein folding, ER dysfunction can lead to chronic ER stress and cell death $(20,23,24)$. Excessive and sustained ER stress can lead to activation of cell death via induction of CHOP, ER-specific caspase 12 , and several other factors $(24,25)$.

The UPR is a cytoprotective response to ER stress. However, failure of the UPR to resolve ER stress plays an important role in several human diseases (20). Recently, we demonstrated that chronic ER stress is associated with TM dysfunction and development of glaucoma in a mouse model of POAG $(26,27)$. We have also shown that expression of mutant myocilin (MYOC) induces ER stress in the TM, which is associated with elevation of IOP in $T g$-MYOC ${ }^{Y 437 H}$ mice. In the present study, we sought to examine whether increased ER stress in the TM is responsible for elevating IOP in other models of glaucoma. We particularly chose to examine glucocorticoid-induced glaucoma because, similar to 

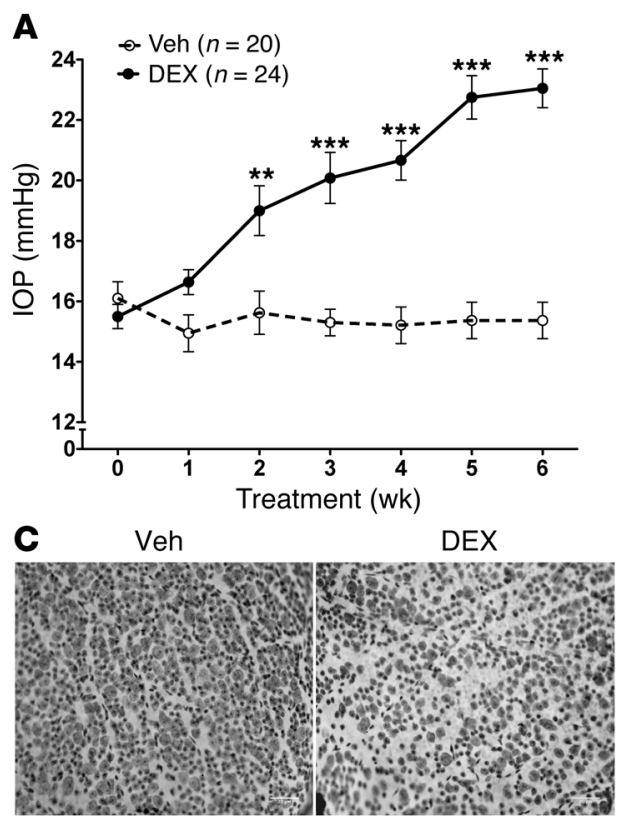

\section{E}

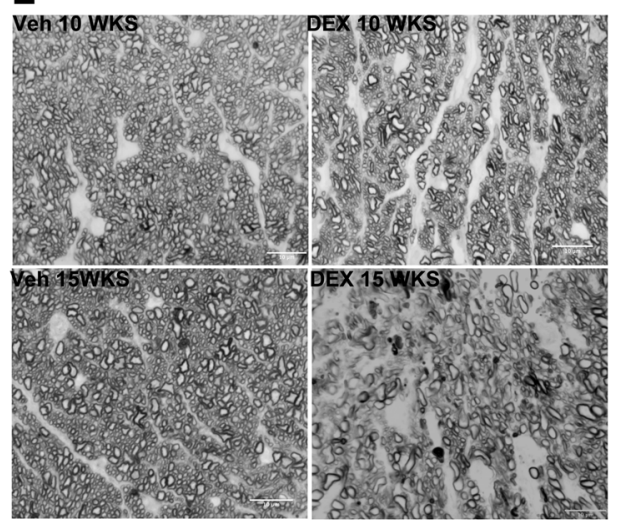

B

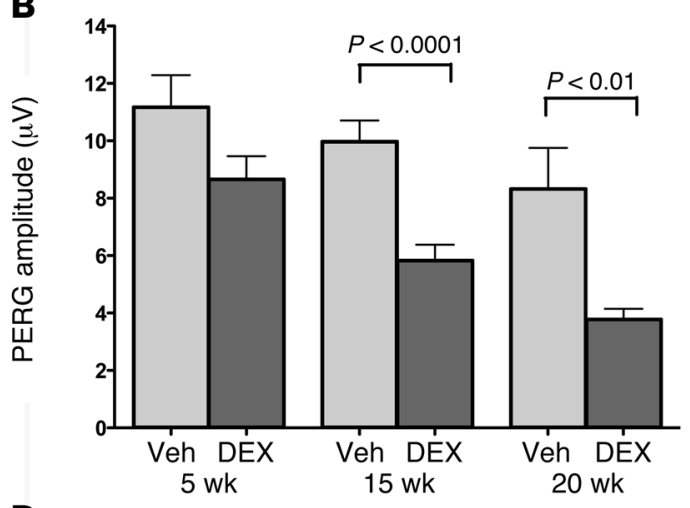

D

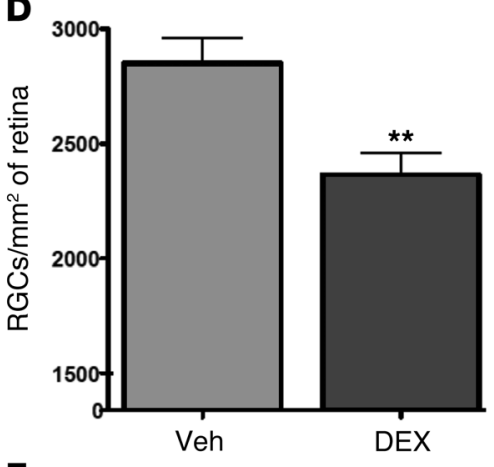

$\mathbf{F}$

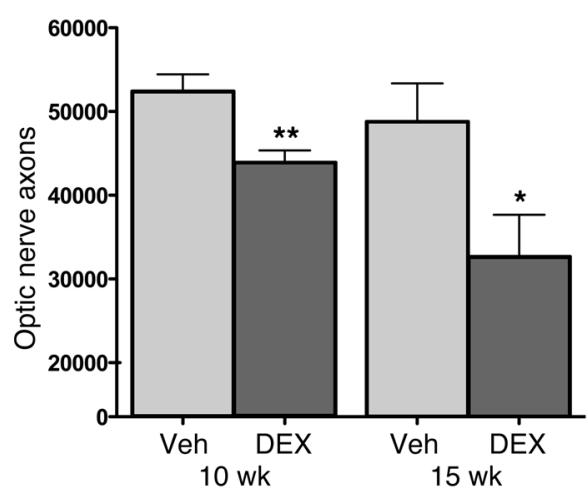

\section{Figure 1}

Topical ocular dexamethasone induces glaucoma in mice. (A) Elevated IOP in dexamethasone-treated C57BL/6 mice. Topical ocular vehicle (sterile PBS) or dexamethasone (0.1\%) was administered 3 times daily for up to 20 weeks. IOP measurements of dexamethasone-treated $(n=20-24)$ and vehicle-treated $(n=20)$ mice are shown from week 0 to 6 of treatment. ${ }^{* \star} P<0.005$, ${ }^{* * *} P<0.0001$, unpaired $t$ test. (B) Progressive RGC functional loss in dexamethasone-treated mice. PERG amplitudes (P50-N95) in vehicle and dexamethasone-treated mice at $5(n=5), 15$ $(n=10)$, and $20(n=6)$ weeks of treatment. (C and D) Loss of RGCs in dexamethasone-treated mice. (C) Representative images of Nissl-stained whole-mount retinas from mice treated for 20 weeks with vehicle or dexamethasone. (D) Remaining cells in ganglion layer were counted in the periphery of the retina. $n=5$ (vehicle); 10 (dexamethasone). ${ }^{* \star} P=0.0045$, unpaired $t$ test. (E and $\mathbf{F}$ ) Progressive optic nerve degeneration in mice treated with dexamethasone for 10 or 15 weeks. Optic nerve sections were stained with PPD (E), and mean axon counts $(\mathbf{F})$ were compared in dexamethasone- $(n=8)$ and vehicle-treated $(n=7-10)$ mice. ${ }^{*} P=0.0174,{ }^{\star \star} P=0.0013$ vs. vehicle, unpaired $t$ test. Scale bar: $10 \mu \mathrm{m}$.

POAG, glucocorticoid-induced ocular hypertension is also caused by increased aqueous humor outflow resistance. Dexamethasone treatment has previously been shown to induce ultrastructural changes in the TM, including the proliferation and activation of the ER and the Golgi apparatus, as well as increased deposition of ECM proteins $(17,28-30)$. Glucocorticoids are also known to cause increased secretory load in the TM, which can induce ER stress, since it overwhelms the ER quality control system (22).
Prednisolone, a synthetic glucocorticoid, has been shown to induce ER stress and activate the UPR in insulin-secreting INS-1E cells (31). Therefore, we hypothesize that glucocorticoids induce ER stress in the TM, which is associated with IOP elevation in glucocorticoid-induced glaucoma.

The objectives of the present study were to develop a murine model of steroid-induced glaucoma and to use this model to investigate the role of ER stress in glucocorticoid-induced ocular hypertension. 


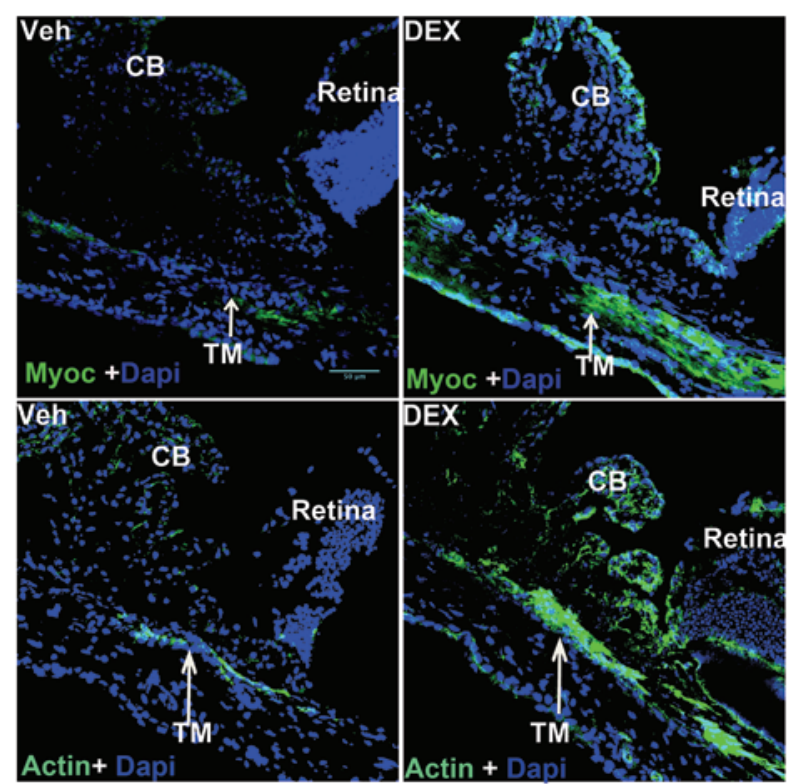

\section{Results}

\section{Topical ocular dexamethasone treatment induces glaucoma} in WT mice

Elevated IOP. To investigate whether glucocorticoids induce ocular hypertension in mice, we applied topical dexamethasone phosphate $(0.1 \%)$ or sterile PBS vehicle eye drops $(-20 \mu \mathrm{l})$ to 3 -month-old C57BL/ 6 mice 3 times per day for 6 weeks. IOP was measured every week. At baseline (no treatment), both treatment groups had similar IOPs (Figure 1A). Although dexamethasone treatment did not alter IOP after 1 week of treatment, starting at 2 weeks, significantly higher IOP was observed in dexamethasone- versus vehicle-treated WT mice, and the difference progressively increased in a linear manner over the course of treatment. The absolute increase in IOP in dexamethasone- versus vehicle-treated mice averaged $3.3 \pm 1.1 \mathrm{mmHg}$ at 2 weeks, $4.7 \pm 1.01 \mathrm{mmHg}$ at 3 weeks, $5.4 \pm 0.91 \mathrm{mmHg}$ at 4 weeks, $7.3 \pm 0.96 \mathrm{mmHg}$ at 5 weeks, and $7.7 \pm 0.84 \mathrm{mmHg}$ at 6 weeks (Figure 1A). Individual IOP measurements revealed that 90\%-95\% of dexamethasone-treated eyes had elevated IOP after 5 weeks of treatment (Supplemental Figure 1; supplemental material available online with this article; doi:10.1172/JCI69774DS1).

We next examined whether topical ocular dexamethasone causes any eye abnormalities. Examination of the anterior segments by slitlamp revealed that dexamethasone- and vehicle-treated eyes were indistinguishable (Supplemental Figure 2). Dexamethasone-treated eyes displayed normal iris and cornea, and none of these mice developed cataract. H\&E staining of the anterior segment revealed that the iridocorneal angle of dexamethasone-treated mice was open. The mice did not have abnormalities of the iris, ciliary body (CB), or cornea and were similar to vehicle-treated mice (Supplemental Figure 3)

Functional loss of RGCs. We next examined whether dexamethasonetreated mice exhibit functional loss of RGCs, as is observed in glaucoma patients. We examined functional deficits in RGCs using pattern electroretinography (PERG), a technique that selectively measures RGC function (Figure 1B). A representative PERG of vehicle- and dexamethasone-treated mice at 20 weeks of treatment illustrates the reduced PERG amplitude (P50-N95) observed with dexamethasone (Supplemental Figure 4). Dexamethasone-treated mice demonstrat-

\section{Figure 2}

Topical ocular dexamethasone increases MYOC and actin levels in the TM. Immunostaining for MYOC and actin merged with nuclear stain DAPI is shown. MYOC and actin were localized to the TM and CB of mice treated with vehicle or dexamethasone for 3 weeks. MYOC and actin levels were increased in TM and $\mathrm{CB}$ of dexamethasone-treated mice $(n=4)$ compared with vehicle-treated mice $(n=3)$. Arrow shows TM. Scale bar: $50 \mu \mathrm{m}$.

ed a 20\% reduction in PERG amplitude compared with vehicle-treated mice at 5 weeks of treatment, with significant further reduction to $40 \%$ at 15 weeks and to $55 \%$ at 20 weeks (Figure 1B).

Loss of ganglion layer cells. We examined whether IOP elevation in dexamethasone-treated mice would lead to RGC loss by performing whole-mount Nissl staining of retinas, as described previously (32). As shown in representative images of Nissl-stained retinas, mice treated for 20 weeks with dexamethasone demonstrated decreased soma number in the ganglion layer (Figure 1C). Quantitation of remaining ganglion layer cells in the periphery of retina revealed that mice treated for 20 weeks with dexamethasone demonstrated significant loss (16\%) of ganglion layer cells compared with vehicle-treated mice (Figure 1D).

Optic nerve degeneration. We also examined optic nerve axonal degeneration by staining optic nerve cross-sections with paraphenylenediamine (PPD) and quantified optic nerve axons. Optic nerve degeneration was evident after dexamethasone treatment (Figure 1E). Dexamethasone-treated mice lost $15 \%$ of axons compared with vehicle-treated mice by 10 weeks of treatment, and $29 \%$ by 15 weeks (Figure 1F), suggestive of progressive neuronal degeneration. We also found that optic nerve area of dexamethasone-treated mice was significantly reduced compared with vehicle-treated mice (reduced $15 \%$ by 10 weeks and $30 \%$ by 15 weeks; Supplemental Figure 5).

\section{Topical dexamethasone increases MYOC, actin, and ECM proteins in the TM}

A hallmark of dexamethasone treatment of the TM is induction of MYOC, actin, and ECM proteins, including fibronectin (FN) (14, 33-36). We next investigated whether topical ocular dexamethasone induces these known biochemical changes in the TM in our mouse model. We performed immunostaining for MYOC and actin on mice treated with vehicle or dexamethasone for 3 weeks (Figure 2). Immunostaining for MYOC revealed a prominent increase in MYOC labeling in the TM of dexamethasone- versus vehicle-treated mice. We also observed a slight increase in MYOC staining in the CB of dexamethasone-treated mice. Similarly, phalloidin, which stains F-actin, revealed a prominent increase in actin in the TM and CB of dexamethasone-treated mice. We also examined whether dexamethasone increases FN. Western blot analysis of the anterior segment tissues from vehicle- and dexamethasonetreated mice showed increased levels of FN with dexamethasone treatment (Supplemental Figure 6).

\section{Dexamethasone induces ER stress and activates the UPR in cultured buman TM cells}

To determine whether dexamethasone induces ER stress in TM cells, we first examined alternative splicing of XBP-1 as a marker for ER stress. Primary human TM cells were treated with dexamethasone (100 nM) for 10 days as described previously (37), and RT-PCR products for XBP 1 were run on $2 \%$ agarose gel. As expected, the unspliced form of XBP-1 (283 bp) was observed in both control and dexameth- 

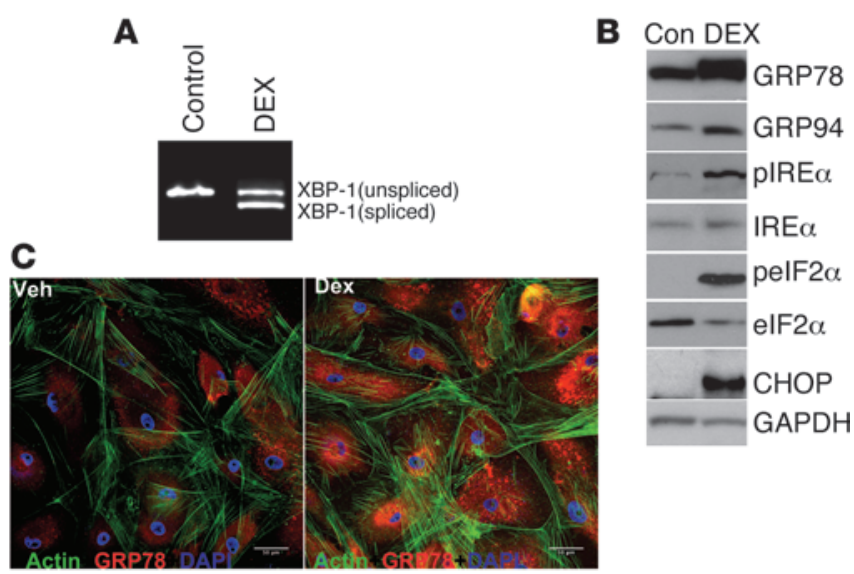

asone-treated TM cells; however, spliced XBP-1 product (257 bp) was observed only in dexamethasone-treated TM cells (Figure 3A).

We next examined ER stress and UPR activation in primary cultures of TM cells obtained from normal human donors by Western blot. Dexamethasone increased ER chaperones (GRP78 and GRP94), induced phosphorylation of IRE1 $\alpha$ and $\operatorname{IF} 2 \alpha$, and increased CHOP protein levels in primary TM cells (Figure 3B). Immunostaining for GRP78 and actin revealed that dexamethasone increased the levels of both in primary TM cells (Figure 3C). Together, these data demonstrated that dexamethasone induces ER stress and activates the UPR in primary TM cells.

\section{Topical ocular dexamethasone induces ER stress and activates the UPR in mouse TM in vivo}

We next sought to examine whether dexamethasone-induced ocular hypertension is associated with induction of ER stress in the anterior segment tissues. Topical dexamethasone treatment did not elevate IOP in WT mice at 1 week of treatment (vehicle, $16.1 \mathrm{mmHg}$; dexamethasone, $15.9 \mathrm{mmHg} ; n=5$ per group). Western blot analysis of the anterior segment tissues obtained from these mice demonstrated that 1 week of dexamethasone treatment increased ER stress and activated the UPR, as evidenced by increased levels of GRP78, ATF-4, cleaved ATF-6, CHOP, and spliced XBP-1 proteins in these tissues (Figure 4A). Dexamethasone also increased MYOC levels in the anterior segment tissues, consistent with previous reports (35).

\section{Figure 3}

Dexamethasone induces ER stress and activates the UPR in human TM cells. (A) Splicing of XBP-1. Primary human TM cells were treated with dexamethasone (100 nM) for 10 days, and total RNA prepared was subjected to RT-PCR with XBP1 primers. The resulting products were subjected to $2 \%$ agarose gel electrophoresis. PCR products were sequenced to further confirm the presence of spliced and unspliced XBP-1. Spliced product was observed at $257 \mathrm{bp}$, while unspliced products were observed at $283 \mathrm{bp}$. Spliced XBP-1 was only observed in dexamethasone-treated samples. (B) Dexamethasone induced ER stress and activated the UPR in human TM cells. Primary human TM cells obtained from normal human donors were treated with dexamethasone for 48 hours. GRP78, GRP94, $\mathrm{CHOP}$, and phosphorylation of IRE1 and elF2 $\alpha$ were examined by Western blot analysis $(n=3)$. (C) Human TM cells were treated with vehicle or dexamethasone for 48 hours and stained with GRP78 and phalloidin (which stains F-actin) $(n=3)$. Dexamethasone treatment increased actin and GRP78 levels. Scale bars: $50 \mu \mathrm{m}$.

These data indicated that topical dexamethasone induces ER stress in WT mice prior to IOP elevation. Dexamethasone also increased GRP78 and CHOP proteins in anterior segment tissues after 8 weeks of treatment (Supplemental Figure 7), indicative of the presence of chronic ER stress. In addition, a prominent increase in GRP78 labeling was detected in the TM of mice treated for 8 weeks with dexamethasone compared with vehicle treatment (Figure 4B). Although a weak signal for GRP78 was detected in the corneal endothelium in vehicle-treated mice, corneal GRP78 did not appear to change in dexamethasone-treated mice. In addition, GRP78 was abundantly present in $\mathrm{CB}$, and its level remained unchanged in dexamethasoneversus vehicle-treated mice (Figure 4B).

\section{Withdrawal of dexamethasone leads to reduction of elevated IOP and ER stress-associated with dexamethasone treatment}

In humans, withdrawal of glucocorticoid in most cases returns elevated IOP to baseline $(18,38)$. Therefore, we examined whether a similar phenomenon occurred in our mouse model. Topical dexamethasone or vehicle eye drops were given to WT mice for 3 weeks. Dexamethasone significantly elevated IOP at 3 weeks of treatment (dexamethasone, $22.4 \pm 0.76 \mathrm{mmHg}$; vehicle, $17.3 \pm 0.67 \mathrm{mmHg}$; $n=10 ; P<0.0001, t$ test). After 3 weeks of treatment, dexamethasone-treated mice were randomly divided into 2 groups: one received topical dexamethasone, and the other vehicle (i.e., dexamethasone withdrawal), for another 2 weeks. Subsequent IOP measurements
A

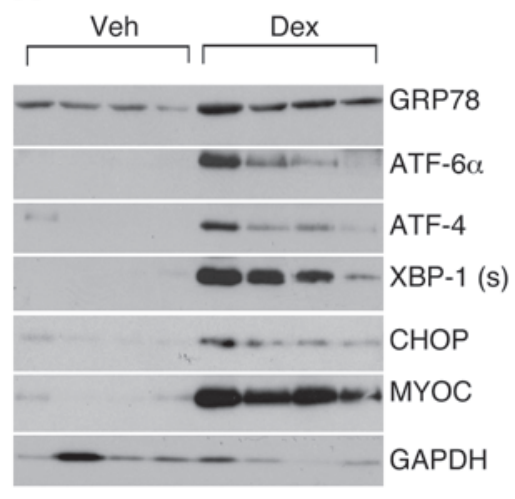

B

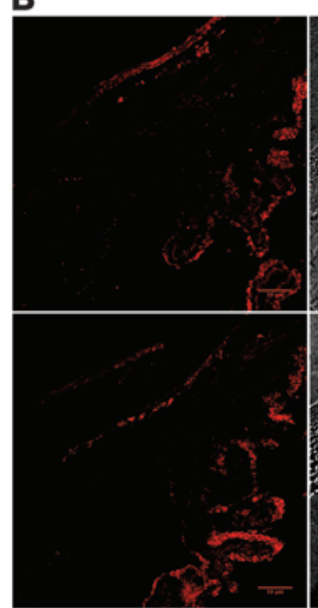

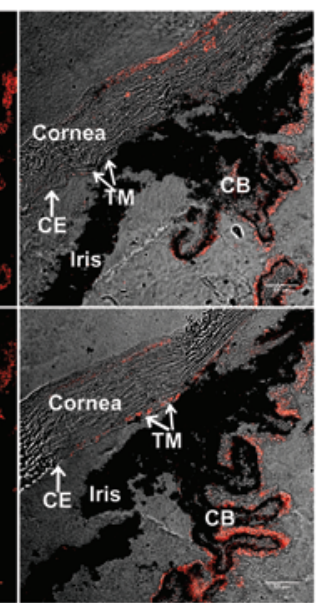

\section{Figure 4}

Topical dexamethasone induces ER stress and activates the UPR in TM tissues of WT mice. (A) GRP78, activated ATF-6 $\alpha$, ATF-4, spliced XBP-1, CHOP, MYOC, and GAPDH (loading control) were examined by Western blot analysis in anterior segment tissues of mice treated for 1 week with vehicle or dexamethasone ( $n=4$ per group) (B) Representative immunostaining for GRP78 in the anterior segment tissues of mice treated for 8 weeks with vehicle or dexamethasone $(n=5$ per group), showing increased GRP78 in the TM (arrows) of dexamethasone-treated mice. CE, corneal endothelium. Scale bars: $50 \mu \mathrm{m}$. 


\section{A}

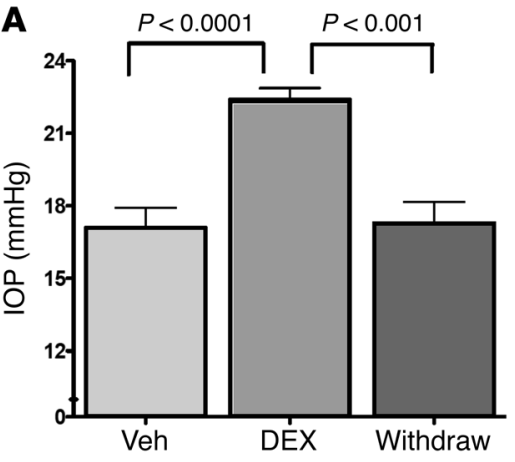

C

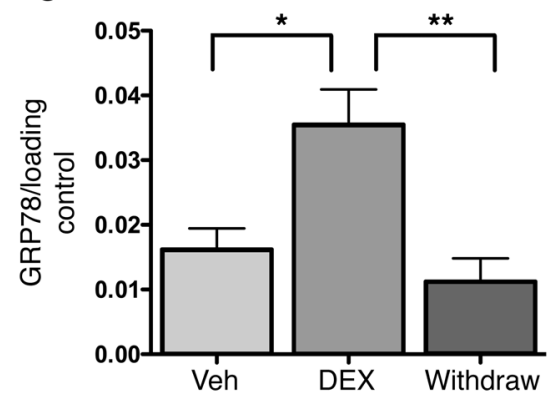

D

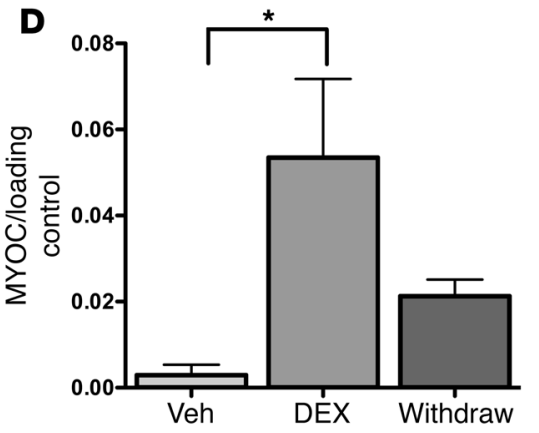

B

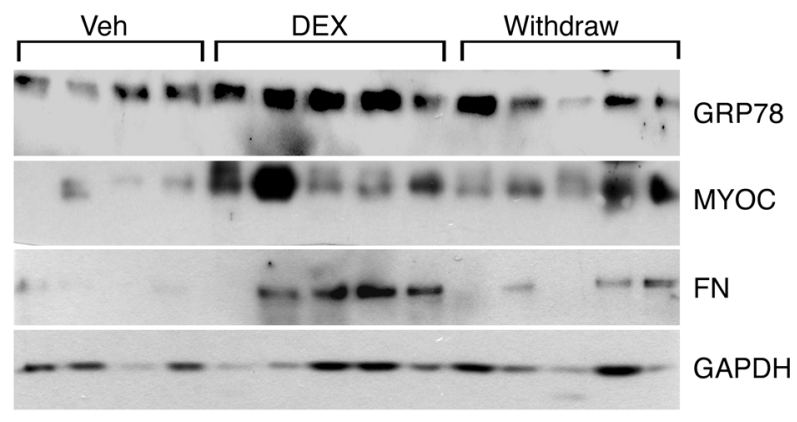

E

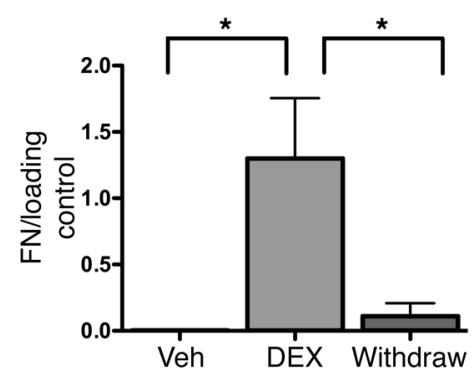

Figure 5

Dexamethasone withdrawal reduces dexamethasone-associated elevations in IOP and ER stress. WT mice were given topical ocular vehicle or dexamethasone for 3 weeks (IOP measurements confirmed elevated IOP with dexamethasone treatment). At this time point, dexamethasonetreated mice were randomly divided into 2 groups: one received topical dexamethasone, and the other vehicle (i.e., dexamethasone withdrawal), for another 2 weeks. (A) 2 weeks of dexamethasone withdrawal significantly reduced IOP ( $n=10$ per group). $P$ values were determined by 1 -way ANOVA. (B) Western blot analysis of GRP78, MYOC, and FN in anterior segment tissue lysates. (C-E) Densitometric analysis of GRP78 (C), MYOC (D), and FN (E) levels, normalized to loading control GAPDH, revealed that dexamethasone withdrawal reduced the dexamethasoneassociated elevations in these proteins. ${ }^{*} P<0.05,{ }^{\star \star} P<0.005$, 1-way ANOVA.

revealed that dexamethasone-withdrawn mice had significantly reduced IOP compared with continued dexamethasone treatment, with levels returning to baseline (Figure 5A). These data indicated that withdrawal of dexamethasone reduces dexamethasone-induced ocular hypertension, similar to the response seen in human patients.

We next examined whether withdrawal of dexamethasone treatment also decreases ER stress. Western blot analysis of anterior segment tissues demonstrated that dexamethasone significantly increased GRP78. However, GRP78 levels were significantly reduced to baseline in dexamethasone-withdrawn mice (Figure 5, $\mathrm{B}$ and $\mathrm{C}$ ). Interestingly, MYOC and FN levels were also reduced after dexamethasone withdrawal (Figure 5, D and E).

\section{Deletion of Chop protects from dexamethasone-induced ocular bypertension}

Induction of $\mathrm{CHOP}$ has been shown to be associated with chronic ER stress. In our model, we observed that dexamethasone treatment increased CHOP levels in the anterior segment tissues, preceding IOP elevation by 1 week (Figure 4A). CHOP levels were also increased at 8 weeks of treatment (Supplemental Figure 7). Deletion of Chop has been shown to be protective against chronic ER stress in multiple studies $(25,39,40)$. Thus, we examined whether deletion of Chop also protects against dexamethasone-induced ocular hypertension. Topical ocular dexamethasone was given to WT and Chop knockout mice (C57BL/6 background) for 3 weeks, and IOP was measured every week. Topical ocular dexamethasone significantly elevated IOP in WT mice after 3 weeks of treatment (dexamethasone,
$23.2 \pm 0.6 \mathrm{mmHg}$; vehicle, $15.8 \pm 0.8 \mathrm{mmHg}$; Figure 6A). However, IOP was not significantly elevated with dexamethasone compared with vehicle treatment in Chop knockout mice (dexamethasone, $18.2 \pm 0.8 \mathrm{mmHg}$; vehicle, $15.7 \pm 0.6 \mathrm{mmHg}$; Figure $6 \mathrm{~A}$ ).

We further examined ER stress markers in the anterior segment tissues of WT and Chop knockout mice. In WT mice, dexamethasone induced ER stress markers, including GRP78 and CHOP along with MYOC (Figure 6B). However, dexamethasone treatment reduced GRP78, ATF-4, and MYOC levels compared with vehicle treatment in Chop knockout mice (Figure 6C). Densitometric analysis revealed that dexamethasone increased GRP78 and MYOC levels 2.5-fold compared with vehicle control in WT mice (Figure 6D). However, dexamethasone-treated Chop knockout mice exhibited significantly decreased GRP78 and MYOC levels compared with dexamethasonetreated WT mice. These data demonstrated that deletion of Chop protects mice from dexamethasone-induced ocular hypertension and also reduces ER stress associated with dexamethasone treatment.

\section{Reduction of ER stress by the chemical chaperone sodium} 4-phenylbutyrate (PBA) prevents IOP elevation by dexamethasone We next sought to examine whether reducing ER stress would rescue dexamethasone-induced ocular hypertension. WT mice were given topical ocular vehicle or dexamethasone for 3 weeks. Dexamethasone-treated mice were divided into 2 groups: one received $20 \mathrm{mM}$ PBA in drinking water, while the other received plain water. IOP measurements after 3 weeks of treatment demonstrated that topical ocular dexamethasone treatment in control mice significantly 


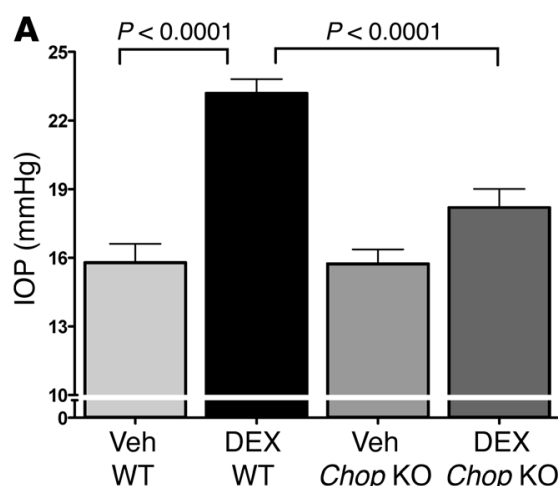

C

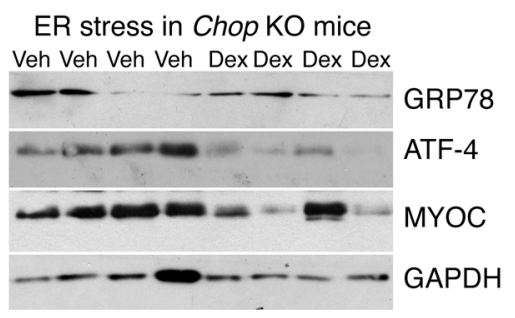

B

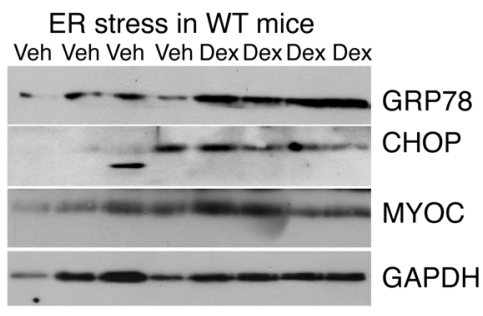

D

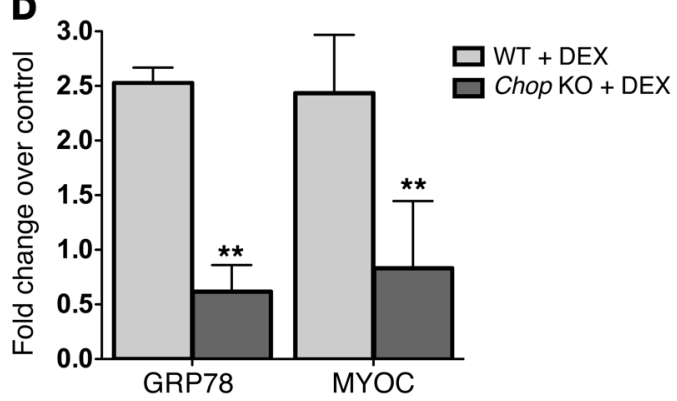

\section{Figure 6}

Deletion of Chop protects against dexamethasone-induced ocular hypertension. (A) Chop knockout protected mice from dexamethasone-induced ocular hypertension. WT and Chop knockout mice were given topical ocular vehicle or dexamethasone for 3 weeks. IOP measurements showed that dexamethasone elevated IOP significantly compared with vehicle in WT mice, but not in Chop knockout mice ( $n=10$ per group). $P$ values were determined by 1-way ANOVA. (B and C) Western blot analysis for GRP78, CHOP, and MYOC in anterior segment tissues of vehicle- and dexamethasone-treated WT (B) and Chop knockout (C) mice ( $n=4$ per group). (D) ER stress and MYOC levels, shown as fold change relative to vehicle control, were reduced with dexamethasone treatment in Chop knockout compared with WT mice. ${ }^{\star \star} P<0.005$, unpaired $t$ test. elevated IOP compared with topical ocular vehicle treatment (dexamethasone, $22.6 \pm 0.5 \mathrm{mmHg}$; vehicle, $16 \pm 0.7 \mathrm{mmHg}$; Figure 7A). Interestingly, when PBA was given to dexamethasone-treated mice, IOP was significantly reduced compared with mice treated with dexamethasone alone (dexamethasone plus PBA, $18.9 \pm 0.5 \mathrm{mmHg}$; dexamethasone, $22.6 \pm 0.5 \mathrm{mmHg}$; Figure 7A). These data demonstrated that systemic PBA prevents IOP elevation by dexamethasone.

We next examined whether PBA reduces ER stress induced by dexamethasone treatment. Western blot and densitometric analyses of ER stress markers in the anterior segment tissues demonstrated that a 3-week treatment with dexamethasone increased levels of the ER stress markers GRP78, spliced XBP-1, and CHOP, which were significantly reduced by PBA treatment (Figure 7, B and C). Interestingly, PBA treatment also decreased the elevated FN levels associated with dexamethasone treatment (Supplemental Figure 8). We further examined the effect of PBA on dexamethasone-induced ER stress using primary human TM cells. TM cells were treated with dexamethasone with or without PBA $(5 \mathrm{mM})$ for 48 hours. Western blot analysis of cell lysates demonstrated that dexamethasone treatment alone increased levels of GRP78, GRP94, MYOC, and phosphorylated eIF $2 \alpha$ in TM cells (Figure 7D). Expression of all these ER stress markers was reduced in TM cells with concomitant PBA treatment. These data demonstrated that PBA prevents dexamethasone-induced ocular hypertension and reduces ER stress associated with dexamethasone treatment in the anterior segment tissues.

\section{Discussion}

Ocular hypertension is a serious side effect of glucocorticoid therapy that can lead to a secondary iatrogenic form of open-angle glaucoma and, if unrecognized, subsequent vision loss. In the present study, we developed a new mouse model of glucocorticoidinduced glaucoma and investigated the molecular mechanisms that lead to ocular hypertension. Topical ocular dexamethasone treatment induced ocular hypertension and resulted in open-angle glaucoma in otherwise healthy $\mathrm{C} 57 \mathrm{BL} / 6$ mice that was similar to steroid glaucoma in human patients. We further demonstrated that dexamethasone induced ER stress in the TM, which was associated with IOP elevation. Withdrawal of dexamethasone treatment reduced dexamethasone-induced elevations in IOP and ER stress. Dexamethasone also induced expression of CHOP, a marker of chronic ER stress, in anterior segment tissues. Deletion of Chop reduced ER stress in the TM and, in turn, protected against glucocorticoid-induced ocular hypertension. Reduction of ER stress by PBA prevented dexamethasone-induced IOP elevation, which further supports the involvement of ER stress in glucocorticoidinduced ocular hypertension. Thus, chronic ER stress appears to play a central role in the development of ocular hypertension in our mouse model of glucocorticoid-induced glaucoma.

Glucocorticoid-induced ocular hypertension has been studied in several animal models, including monkeys (41), cows (42), cats (43), rabbits (44), sheep (45), rats (46), and recently mice (47). However, a model of glucocorticoid-induced glaucoma has not previously been developed. A recent study by Whitlock and colleagues demonstrated that systemic dexamethasone administration elevated IOP by 3-4 mmHg in hybrid mice (47). In our present study, topical ocular dexamethasone elevated the IOP of normal C57BL/6J mice by $7 \mathrm{mmHg}$. In addition to developing ocular hypertension, chronic dexamethasone treatment caused RGC structural and functional loss, as well as optic nerve degeneration, similar to that observed in patients with glucocorticoid-induced glaucoma. In our model, as in the human disorder, elevated IOP preceded PERG abnormalities: examination of RGC function by PERG at 5 weeks (2-3 weeks after elevated IOP) showed a nonsignificant reduction in PERG amplitudes in dexamethasone-treated mice. Thus, it is unlikely that dexamethasone leads to RGC loss within 2-3 weeks prior to IOP elevation in our mouse model. Withdrawal of dexamethasone treatment normalized mouse IOP to baseline levels, similar to human patients. Consistent with previously reported studies, dexamethasone induced similar biochemical changes in the TM in our mouse model of glucocorticoid-induced glaucoma, including increased 
A

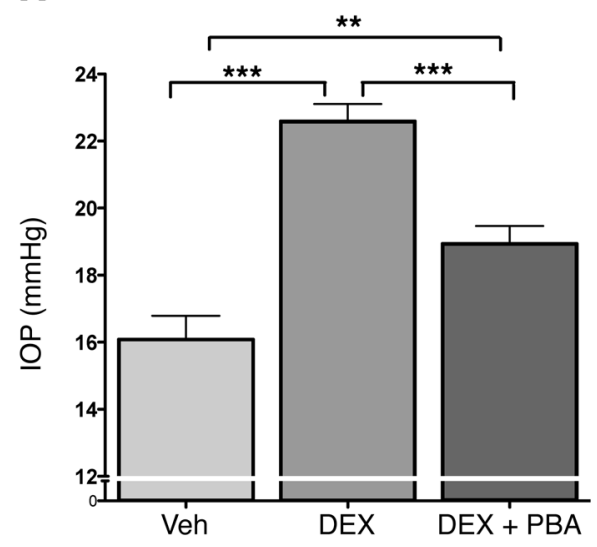

C

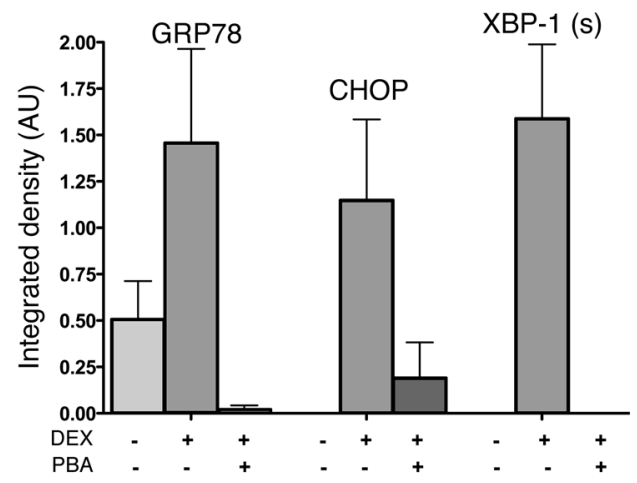

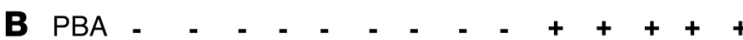

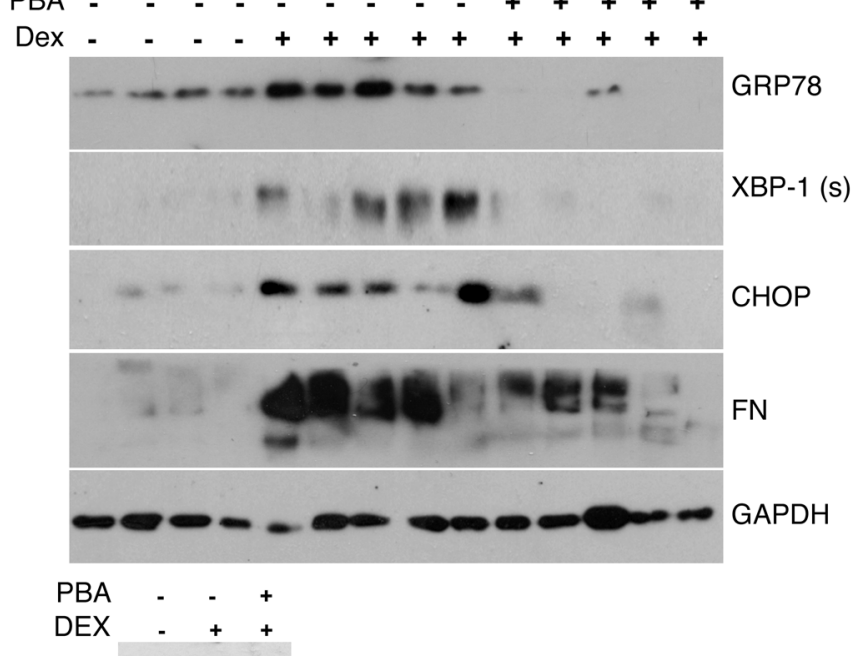

GRP78

GRP94

pelF2- $\alpha$

elF2- $\alpha$

MYOC

GAPDH

\section{Figure 7}

Decreasing ER stress by administration of the chemical chaperone PBA reduces IOP elevation by dexamethasone. (A-C) WT mice were given topical ocular vehicle or dexamethasone for 3 weeks. Dexamethasone-treated mice were divided into 2 groups: one received water, the other received $20 \mathrm{mM}$ PBA in drinking water. (A) PBA treatment significantly protected from dexamethasone-induced IOP elevation $\left(n=20\right.$ per group). ${ }^{* *} P<0.05$, ${ }^{* * *} P<0.005$, 1-way ANOVA. (B and C) Western blot (B) and densitometric analysis (C) of ER stress markers in anterior segment tissues revealed that combined dexamethasone and PBA treatment reduced ER stress markers compared with dexamethasone treatment alone ( $n=5$ per group). (D) PBA reduced ER stress associated with dexamethasone in human TM cells. Human TM cells were treated with dexamethasone with or without 5 mM PBA. Total cell lysates were subjected to Western blot analysis for GRP78, GRP94, phosphorylated and total eIF2 $\alpha$, MYOC, and GAPDH.

MYOC, actin, and FN. These data indicated that our mouse model appropriately mimics human glucocorticoid-induced glaucoma.

Glucocorticoids induce ocular hypertension in a subset of the human population $(10,12)$. Differential glucocorticoid responsiveness is also observed in several animal models, including nonhuman primate eyes (41), rabbits (44), mice (47), and the bovine perfusion cultured anterior segment model (48). In contrast, a $100 \%$ responder rate was reported in cattle in vivo (42). Whitlock and colleagues systemically administered dexamethasone to hybrid mice (B6:129) and showed increased IOP in approximately one-half of the mice (47). In our present study, $90 \%-95 \%$ of mice treated with dexamethasone developed ocular hypertension after 5 weeks of treatment in our genetically homogeneous pure $\mathrm{C} 57 \mathrm{BL} / 6$ strain. These findings suggest that genetic heterogeneity may be responsible for the differential glucocorticoid responsiveness. Several studies have suggested that differences in the levels of alternatively spliced glucocorticoid receptor isoforms regulate glucocorticoid responsiveness in TM cells $(37,49)$.

Our data demonstrated that induction of ER stress in the TM was associated with dexamethasone-induced ocular hypertension.
Specifically, ER stress induction preceded IOP elevation. ER stress was also chronically elevated over the course of treatment. Cessation of dexamethasone treatment returned elevated IOP to baseline and also decreased ER stress associated with dexamethasone treatment in anterior segment tissues. Furthermore, knockout of Chop or treatment with PBA protected mice from dexamethasone-induced ocular hypertension, along with reducing ER stress in the anterior segment. It is not entirely clear how dexamethasone induces ER stress in the TM. Dexamethasone is known to increase the overall protein secretory load, which can lead to ER stress. In our model, dexamethasone increased MYOC, $\mathrm{FN}$, and actin in primary TM cells as well as in the mouse TM. We hypothesize that increased protein processing and accumulation of these proteins in the TM may result in ER stress in our model of glucocorticoid-induced ocular hypertension. Consistent with this hypothesis, our results showed that increased levels of MYOC, actin, and FN were associated with dexamethasone-induced ocular hypertension. Specifically, withdrawal of dexamethasone treatment decreased MYOC and FN levels in the anterior segment. In addition, Chop knockout mice were protected from dexamethasone-induced IOP elevation and also had reduced levels of MYOC 
and markers of ER stress in the anterior segment tissues. Furthermore, treatment with the chemical chaperone PBA reduced elevated IOP and decreased FN, MYOC, and ER stress in the TM. Together, these findings suggest that induction of ER stress associated with increased levels of MYOC and ECM proteins may cause dysfunction of the TM, resulting in IOP elevation in glucocorticoid-induced glaucoma. Our present findings provide new directions for understanding glucocorticoid-induced ocular hypertension.

This study demonstrated a prominent increase in GRP78, MYOC, and actin levels in the TM. It is widely accepted that biochemical and morphological changes in the TM are responsible for increased TM outflow resistance and ocular hypertension in steroid-induced glaucoma (14). Consistent with this, Kumar et al. recently described a new model of steroid-induced changes in mouse outflow facility and demonstrated that steroid treatment reduces outflow facility, despite no noticeable change in IOP (50). In addition to our findings in the TM, we observed increased immunostaining for MYOC and actin in the CB of dexamethasone-treated WT mice; thus, we cannot exclude a potential role for the $\mathrm{CB}$ in ocular hypertension.

Several studies have shown that induction of Chop is associated with cellular dysfunction and that its overexpression leads to ER stress-mediated cell death (25). We observed that dexamethasone induced Chop over the course of treatment. Interestingly, deletion of Chop protected mice from dexamethasone-induced ocular hypertension and also reduced ER stress compared with WT mice. CHOP may theoretically cause TM dysfunction and/or TM cell death, thus resulting in IOP elevation. TUNEL staining of the anterior chamber did not show apoptotic TM cell death (Supplemental Figure 9), suggestive of a nonapoptotic role of CHOP in our mouse model of steroid glaucoma. Deletion of Chop has previously been shown to protect against ER stress by decreasing ER client protein load and changing redox conditions (51). Deletion of Chop improves $\beta$ cell function and protects from ER stress by enhancing UPR and oxidative stress response genes (39). Chop deletion may also prevent glucocorticoid-induced dysfunction of the TM and prevent ocular hypertension by acting upstream of the ER stress pathway. It is also possible that Chop deletion enhances ER function in handling misfolded proteins and overall secretory pathway function, thereby protecting TM cells from the consequences of protein misfolding. Consistent with this hypothesis, we observed reduced levels of MYOC and ER stress in dexamethasone-treated Chop knockout mice compared with vehicle-treated Chop knockout and dexamethasonetreated WT mice. Similarly, PBA treatment reduced dexamethasoneinduced MYOC and FN levels, which in turn decreased ER stress and prevented IOP elevation. Alternatively, Chop deletion may inhibit the progression of stressed TM cells to apoptosis and activate a positive feedback mechanism that decreases ER stress directly in TM tissues.

Steroid responsiveness is higher in POAG patients and their descendants. However, steroid responsiveness in MYOC patients has not been studied. Because glucocorticoids induce WT MYOC expression, it was originally hypothesized that increased MYOC accumulates in the TM, leading to increased outflow resistance and IOP elevation in glucocorticoid glaucoma. However, transgenic mice overexpressing WT MYOC do not develop glaucoma, which suggests that overexpression of MYOC is not in itself sufficient to cause glaucoma in mice (52). It is possible that dexamethasone alters WT MYOC processing in the ER, thus reducing its secretion and inducing ER stress in the TM. Since induction of ER stress is associated with elevation of IOP in $\mathrm{Tg}-M Y O C^{Y 437 H}$ mice, which express mutant MYOC, it is conceivable that dexamethasone treatment may worsen the glaucoma phenotypes of Tg-MYOC ${ }^{Y 437 H}$ mice. Specifically, dexamethasone increases WT MYOC, which can interact with mutant MYOC (53). These events can lead to increased MYOC aggregates in the ER of the TM, thus worsening the glaucoma in $\mathrm{Tg}-M Y O C^{Y 437 H}$ mice. Future studies will be aimed at understanding the role of WT and mutant MYOC in glucocorticoid-associated glaucoma.

Systemic PBA treatment prevented glucocorticoid-induced IOP elevation by reducing ER stress in the anterior segment. Previously, we demonstrated that topical PBA rescued glaucoma in Tg-MYOC ${ }^{Y 437 H}$ mice by reducing mutant MYOC accumulation and facilitating MYOC secretion in the aqueous humor, consequently reducing ER stress in the TM $(26,27)$. Thus, it is conceivable that PBA may prevent ER stress and IOP elevation in glucocorticoid-induced ocular hypertension by similar mechanisms, including decreasing ER load, enhancing protein secretion, and reducing protein accumulation in the TM. Consistent with this hypothesis, dexamethasone-induced MYOC and FN levels were significantly decreased to baseline levels by PBA treatment. It is unlikely that PBA has a direct effect on MYOC transcript levels, since PBA has been shown to increase MYOC secretion within 30 minutes of treatment (54), suggestive of a direct effect of PBA on the secretory pathway.

In conclusion, we here established a mouse model of glucocorticoid-induced glaucoma and demonstrated that ER stress played a critical role in glucocorticoid-induced ocular hypertension. Furthermore, our demonstration of reduced ER stress by chemical chaperones such as PBA provides a promising potential treatment for glaucoma. These studies provide new mechanistic insights into glucocorticoid-induced ocular hypertension and a novel target for treatment of glucocorticoid-induced glaucoma.

\section{Methods}

Mouse husbandry. C57BL/6J mice were obtained from the Jackson Laboratory. Mice were housed and bred at the University of Iowa as described previously (26).

Topical ocular dexamethasone and vehicle treatment. 4 independent experiments containing at least 10 mice per group were performed to evaluate the effect of topical ocular dexamethasone administration. Topical $0.1 \%$ dexamethasone phosphate (Bausch \& Lomb Inc.) was used. Sterile PBS was used as a vehicle eye drop. 3-month-old C57BL/6J mice were given topical ocular $0.1 \%$ dexamethasone phosphate or sterile PBS (vehicle) eye drops 3 times daily for up to 20 weeks. A small eye drop $(20 \mu \mathrm{l})$ was applied to both eyes. The initial daily dose was given between $9 \mathrm{Am}$ and $10 \mathrm{Am}$, the second dose between 1 PM and 2 PM, and the third dose between 6 PM and 7 PM. To ensure the effective penetration of the eye drops into the anterior chamber, mice were lightly held for 30-40 seconds after drop administration, then released in their cages. Chop knockout mice (Jackson Labs) were obtained from the laboratory of T. Rutworski (University of Iowa). For dexamethasone withdrawal, WT mice were given topical vehicle or dexamethasone for 3 weeks. At 3 weeks, the dexamethasone-treated mice were randomly divided into 2 groups: one received topical dexamethasone, and the other vehicle, for another 2 weeks. IOP was measured during this period, and anterior segment tissues were isolated for Western blot analysis of ER stress.

IOP measurements. IOP was measured with a rebound tonometer, as previously described (26). In brief, mice were acclimated to the procedure room and anesthetized with $2.5 \%$ isoflurane plus $100 \%$ oxygen. IOP was measured with a tonometer (TonoLab; Colonial Medical Supply). All IOP cohorts included male and female mice. Daytime IOP was measured between $10 \mathrm{AM}$ and $2 \mathrm{PM}$. IOP was measured every week for several weeks in each group of mice. Mice were anesthetized with isoflurane for very short times (approximately 2 minutes); longer isoflurane exposure rapidly 
decreased IOP, especially in dexamethasone-treated mice. IOP measurements were performed in a masked manner by a technician uninformed of treatment group. Because each eye responds differently to treatment, we considered each eye measurement as an independent sample.

PERG. PERG was used to objectively measure the function of RGCs by recording amplitudes and latency of N35-P50 and P50-N95 PERG waveforms, as described previously (26).

Retina staining. Nissl staining of retinal whole mounts was used to determine the number of cells within the RGC layer, as described previously (32). Mice were euthanized, and eyes were enucleated and fixed in $4 \%$ paraformaldehyde in PBS buffer for 3 hours. After rinsing the eyeball in PBS, the anterior chamber was removed, and the eyecup was incubated in PBS containing $0.3 \%$ Triton X-100 overnight at room temperature. The retina was then isolated, mounted with the ganglion cell layer up on a glass slide, and flattened under a coverslip with a 10 -g weight on top. Coverslips were removed, and air-dried retinas were stained with $1 \%$ cresyl violet acetate (Nissl stain, in $0.25 \%$ acetic acid) by brush-painting, then dehydrated in $100 \%$ ethanol, cleared in xylene, and finally mounted and coverslipped.

Quantitation of remaining ganglion layer cells was performed by counting soma in the periphery of the retina. 16 nonoverlapping images covering most of the periphery of retina (4 per quadrant) were taken at $\times 400$ magnification, and cells were counted in the area equal to $10 \%$ of the total retinal cross-sectional area. The number of remaining cells per square millimeter are represented graphically in Figure 1D.

Assessment of optic nerve damage. Optic nerve neurodegeneration was examined using PPD-stained optic nerve cross-sections, as described previously (26).

TM cell cultures and dexamethasone treatment. Human TM cells were grown as described previously (37). Briefly, TM cells were grown in DMEM containing L-glutamine $(0.292 \mathrm{mg} / \mathrm{ml}$; Invitrogen $)$, penicillin $(100 \mathrm{U} / \mathrm{ml})$ and streptomycin ( $0.1 \mathrm{mg} / \mathrm{ml}$; Invitrogen), and 10\% FBS (Invitrogen). For treatment, TM cells were incubated with fresh medium with or without $100 \mathrm{nM}$ dexamethasone (Sigma-Aldrich) for 10 days. Total RNA was isolated and subjected to RT-PCR of XBP1. For Western blot analysis, TM cells were treated with dexamethasone $(500 \mathrm{nM})$ for 48 hours, and total cell lysates were subjected to Western blot analysis of ER stress markers. For PBA treatment, TM cells were grown in dexamethasone $(500 \mathrm{nM})$ with or without PBA $(5 \mathrm{mM})$ for 48 hours. Total cell lysates were collected and subjected to Western blot analysis, as described previously (26).

Measurement of XBP-1 splicing. XBP1 mRNA was amplified using primers $5^{\prime}$-AACTCCAGCTAGAAAATCAGC-3' and $5^{\prime}$-CCATGGGAAGATGTTCTGGG-3'. PCR was done for 25 cycles $\left(94^{\circ} \mathrm{C}, 30\right.$ seconds; $55^{\circ} \mathrm{C}$, 30 seconds; $72^{\circ} \mathrm{C}, 30$ seconds [ 2 minutes in the final cycle]). Fragments representing spliced ( $257 \mathrm{bp}$ ) and unspliced (283 bp) XBP-1 were run on $2 \%$ agarose gels as described previously (55). Spliced and unspliced bands were further excised and sequenced to confirm splicing of XBP-1.

Immunostaining. Mouse anterior segments were fixed in $4 \%$ formaldehyde and embedded in OCT. Sections were then blocked with $5 \%$ normal serum. Slides were incubated overnight with primary antibody (1:250) and washed 3 times with PBS, followed by a 2-hour incubation with appropriate Alexa Fluor secondary antibodies (1:200; Invitrogen). Sections were subsequently incubated with DAPI for 30 minutes to stain nuclei, washed, and then mounted. Images were captured using a Zeiss 710 confocal imaging system at the University of Iowa Central Microscopy Research Facility. The MYOC antibody used for immunostaining was obtained from S. Tomarev (NIH). GRP78 was obtained from Gene Tex (1:250). Donkey anti-goat (1:500) and phalloidin stain (1:500) were obtained from Invitrogen.

Western blot analysis. Anterior segment tissues were carefully dissected and lysed in RIPA lysis buffer, as previously described (26). All samples were obtained and processed at the same time. Since Western blot analysis was performed on tissues of the anterior segment, which contains a small amount of cornea, iris, and $\mathrm{CB}$ and the entire $\mathrm{TM}$, it is possible that small variation in dissected tissues may change protein levels in each sample. However, it should be noted that total cumulative response shown by densitometric analysis more accurately demonstrates the overall pattern of response. Considering the small size of anterior segment tissues, total protein lysates from each eye only allowed a few Western blot analyses. Therefore, we focused on analysis of the selected ER stress markers GRP78, ATF-4, and CHOP in most subsequent studies. We established induction of ER stress by dexamethasone in Figures 3 and 4 using several ER stress markers, including phosphorylation of IRE1 $\alpha$ and eIF $2 \alpha$ and increased levels of GRP78, GRP94, cleaved ATF-6, ATF-4, and CHOP. To ensure equal protein loading, the same blot was subsequently incubated with a GAPDH monoclonal antibody (Cell Signaling Technology Inc.). Quantitation was done using Image J software. Antibodies for GRP94, phosphorylated and total eIF $2 \alpha$, and CHOP were purchased from Cell Signaling Technology Inc. Antibody for ATF-6 was purchased from Imagenex Corp. GRP78, MYOC (catalog no. sc137233), and spliced XBP-1 antibodies were purchased from Santa Cruz Biotechnology Inc. Phosphorylated IRE $\alpha$ antibody was obtained from GeneTex (catalog no. GTX63722). Total IRE $\alpha$ antibody was obtained from Santa Cruz Biotechnology Inc.

PBA treatments. 3-month-old WT littermates $(n=30)$ were divided into 3 groups: group 1 was treated with topical vehicle eye drops, group 2 was given dexamethasone eye drops, and group 3 was given dexamethasone eye drops along with $20 \mathrm{mM}$ PBA in drinking water. Pharmaceutical-grade PBA sodium salt was purchased from Scandinavian Formulas, and treatments were conducted as described previously (26). PBA is soluble in water, and thus was added freshly to the drinking water every week for 5 weeks. Water intake measurements were made; treated and untreated mice consumed equal amounts. Mice drank an average of $3 \mathrm{ml}$ of water containing the drug every day. IOP measurements were performed every week. After 5 weeks of treatment, mice were sacrificed, and anterior segment tissues were examined for ER stress markers.

Mouse slit-lamp examination. Anterior chamber phenotypes were assayed with a slit-lamp (SL-D7; Topcon) and photodocumented with a digital camera (D100; Nikon), as described previously (26).

Statistics. All data are presented as mean \pm SEM. For comparisons between 2 groups, unpaired 1-tailed Student's $t$ test was used. For comparisons among 3 or more groups, 1-way ANOVA with Bonferroni multiple-comparison test was used. A $P$ value less than 0.05 was considered significant.

Study approval. All animal procedures performed in this study complied with the ARVO Statement for the Use of Animals in Ophthalmic and Vision Research and were approved by the University of Iowa Animal Care and Use Committee.

\section{Acknowledgments}

This work was supported by funding from the National Eye Institute (grants R01 EY10564 to V.C. Sheffield, K99 EY022077 to G.S. Zode, and 2R01EY016242 to A.F. Clark). V.C. Sheffield is investigator of the Howard Hughes Medical Institute. The authors acknowledge Alcon Labs and the Knights Templar Eye Foundation for providing financial assistance for some experiments. The authors thank Joseph Conner Peter and Allen Choi for assistance in some experiments, Adam Hedberg for technical help with slitlamp analysis, and Matthew Harper for help with PERG.

Received for publication October 7, 2013, and accepted in revised form January 30, 2014.

Address correspondence to: Val C. Sheffield, 4181 Merf, Iowa City, Iowa 52242, USA. Phone: 319.335.6898; Fax: 319.335.7588; E-mail: val-sheffield@uiowa.edu. Or to: Gulab S.Zode, Department of Cell 
Biology and Immunology and North Texas Eye Research Institute, University of North Texas Health Science Center at Fort Worth, 3500 Camp Bowie Blvd., Fort Worth, Texas 76107, USA. Phone: 817.735.0360; Fax: 817.735.2637; E-mail: gulab.zode@unthsc.edu.
Gulab S. Zode's present address is: Department of Cell Biology and Immunology and North Texas Eye Research Institute, University of North Texas Health Science Center at Fort Worth, Fort Worth, Texas, USA.
1. Quigley HA. Number of people with glaucoma worldwide. BrJ Ophthalmol. 1996;80(5):389-393.

2. Quigley HA. Neuronal death in glaucoma. Prog Retin Eye Res. 1999;18(1):39-57.

3. Quigley HA, Broman AT. The number of people with glaucoma worldwide in 2010 and 2020. Br J Ophthalmol. 2006;90(3):262-267.

4. Kwon YH, Fingert JH, Kuehn MH, Alward WL. Primary open-angle glaucoma. N Engl J Med. 2009; 360(11):1113-1124.

5. Libby RT, Gould DB, Anderson MG, John SW. Complex genetics of glaucoma susceptibility. Annu Rev Genomics Hum Genet. 2005;6:15-44.

6. Leske MC, et al. Factors for glaucoma progression and the effect of treatment: the early manifest glaucoma trial. Arch Ophthalmol. 2003;121(1):48-56.

7. Gordon MO, et al. The Ocular Hypertension Treatment Study: baseline factors that predict the onset of primary open-angle glaucoma. Arch Ophthalmol. 2002;120(6):714-720.

8. Rohen JW, Lutjen-Drecoll E, Flugel C, Meyer M, Grierson I. Ultrastructure of the trabecular meshwork in untreated cases of primary open-angle glaucoma (POAG). Exp Eye Res. 1993;56(6):683-692.

9. Bill A. Editorial: The drainage of aqueous humor. Invest Ophthalmol. 1975;14(1):1-3.

10. Armaly MF, Becker B. Intraocular pressure response to topical corticosteroids. Fed Proc. 1965; 24(6):1274-1278.

11. Becker B. Intraocular pressure response to topical corticosteroids. Invest Ophthalmol. 1965;4(2):198-205.

12. Becker B, Mills DW. Corticosteroids and intraocular pressure. Arch Ophthalmol. 1963;70:500-507.

13. Bernstein HN, Mills DW, Becker B. Steroid-induced elevation of intraocular pressure. Arch Ophthalmol. 1963;70:15-18.

14. Clark AF, Wordinger RJ. The role of steroids in outflow resistance. Exp Eye Res. 2009;88(4):752-759.

15. Kersey JP, Broadway DC. Corticosteroid-induced glaucoma: a review of the literature. Eye (Lond). 2006; 20(4):407-416.

16. Jones R, Jones R 3rd, Rhee DJ. Corticosteroidinduced ocular hypertension and glaucoma: a brief review and update of the literature. Curr Opin Ophthalmol. 2006;17(2):163-167.

17. Wordinger RJ, Clark AF. Effects of glucocorticoids on the trabecular meshwork: towards a better understanding of glaucoma. Prog Retin Eye Res. 1999; 18(5):629-667.

18. Armaly MF. Effect of corticosteroids on intraocular pressure and fluid dynamics. II. The effect of dexamethasone in the glaucomatous eye. Arch Ophthalmol. 1963;70:492-499.

19. Schroder M, Kaufman RJ. The mammalian unfolded protein response. Annu Rev Biochem. 2005;74:739-789.

20. Yoshida H. ER stress and diseases. FEBS J. 2007; 274(3):630-658.

21. Yoshida H. [Molecular biology of the ER stress response]. Seikagaku. 2004;76(7):617-630.

22. Marciniak SJ, Ron D. Endoplasmic reticulum stress signaling in disease. Physiol Rev. 2006; 86(4):1133-1149.

23. Zinszner $\mathrm{H}$, et al. CHOP is implicated in programmed cell death in response to impaired function of the endoplasmic reticulum. Genes Dev. 1998;
12(7):982-995.

24. Yamaguchi H, Wang HG. CHOP is involved in endoplasmic reticulum stress-induced apoptosis by enhancing DR5 expression in human carcinoma cells. J Biol Chem. 2004;279(44):45495-45502.

25. Oyadomari S, Mori M. Roles of CHOP/GADD153 in endoplasmic reticulum stress. Cell Death Differ. 2004;11(4):381-389.

26. Zode GS, et al. Reduction of ER stress via a chemical chaperone prevents disease phenotypes in a mouse model of primary open angle glaucoma. J Clin Invest. 2011;121(9):3542-3553.

27. Zode GS, et al. Topical ocular sodium 4-phenylbutyrate rescues glaucoma in a myocilin mouse model of primary open angle glaucoma. Invest Ophthalmol Vis Sci. 2012;53(3):1557-1565.

28. Rohen JW, Linner E, Witmer R. Electron microscopic studies on the trabecular meshwork in two cases of corticosteroid-glaucoma. Exp Eye Res. 1973; 17(1):19-31.

29. Johnson D, Gottanka J, Flugel C, Hoffmann F, Futa R, Lutjen-Drecoll E. Ultrastructural changes in the trabecular meshwork of human eyes treated with corticosteroids. Arch Ophthalmol. 1997; 115(3):375-383.

30. Stamer WD, Hoffman EA, Kurali E, Krauss AH. Unique response profile of trabecular meshwork cells to the novel selective glucocorticoid receptor agonist, GW870086X. Invest Ophthalmol Vis Sci. 2013; 54(3):2100-2107.

31. Linssen MM, et al. Prednisolone-induced $\beta$ cell dysfunction is associated with impaired endoplasmic reticulum homeostasis in INS-1E cells. Cell Signal. 2011;23(11):1708-1715.

32. Anderson MG, Libby RT, Gould DB, Smith RS, John SW. High-dose radiation with bone marrow transfer prevents neurodegeneration in an inherited glaucoma. Proc Natl Acad Sci US A. 2005;102(12):4566-4571.

33. Clark AF, et al. Dexamethasone alters F-actin architecture and promotes cross-linked actin network formation in human trabecular meshwork tissue. Cell Motil Cytoskeleton. 2005;60(2):83-95.

34. Steely HT, Browder SL, Julian MB, Miggans ST, Wilson KL, Clark AF. The effects of dexamethasone on fibronectin expression in cultured human trabecular meshwork cells. Invest Ophthalmol Vis Sci. 1992; 33(7):2242-2250.

35. Clark AF, et al. Glucocorticoid induction of the glaucoma gene MYOC in human and monkey trabecular meshwork cells and tissues. Invest Ophthalmol Vis Sci. 2001;42(8):1769-1780.

36. Shepard AR, Jacobson N, Fingert JH, Stone EM, Sheffield VC, Clark AF. Delayed secondary glucocorticoid responsiveness of MYOC in human trabecular meshwork cells. Invest Ophthalmol Vis Sci. 2001; 42(13):3173-3181.

37. Jain A, Wordinger RJ, Yorio T, Clark AF. Spliceosome protein (SRp) regulation of glucocorticoid receptor isoforms and glucocorticoid response in human trabecular meshwork cells. Invest Ophthalmol Vis Sci. 2012;53(2):857-866.

38. Sihota R, Konkal VL, Dada T, Agarwal HC, Singh R. Prospective, long-term evaluation of steroidinduced glaucoma. Eye. 2008;22(1):26-30.

39. Song B, Scheuner D, Ron D, Pennathur S,
Kaufman RJ. Chop deletion reduces oxidative stress, improves beta cell function, and promotes cell survival in multiple mouse models of diabetes. J Clin Invest. 2008;118(10):3378-3389.

40. Ariyama Y, et al. Chop-deficient mice showed increased adiposity but no glucose intolerance. Obesity (Silver Spring). 2007;15(7):1647-1656.

41. Fingert JH, et al. Evaluation of the myocilin (MYOC) glaucoma gene in monkey and human steroid-induced ocular hypertension. Invest $O p h$ thalmol Vis Sci. 2001;42(1):145-152.

42. Gerometta R, et al. Steroid-induced ocular hypertension in normal cattle. Arch Ophthalmol. 2004; 122(10):1492-1497.

43. Zhan GL, Miranda OC, Bito LZ. Steroid glaucoma: corticosteroid-induced ocular hypertension in cats. Exp Eye Res. 1992;54(2):211-218.

44. Lorenzetti OJ. Effects of corticosteroids on ocular dynamics in rabbits. J Pharmacol Exp Ther. 1970; 175(3):763-772.

45. Gerometta R, Podos SM, Danias J, Candia OA. Steroid-induced ocular hypertension in normal sheep. Invest Ophthalmol Vis Sci. 2009;50(2):669-673.

46. Sawaguchi K, Nakamura Y, Nakamura Y, Sakai $\mathrm{H}$, Sawaguchi S. Myocilin gene expression in the trabecular meshwork of rats in a steroid-induced ocular hypertension model. Ophthalmic Res. 2005; 37(5):235-242.

47. Whitlock NA, McKnight B, Corcoran KN, Rodriguez LA, Rice DS. Increased intraocular pressure in mice treated with dexamethasone. Invest Ophthalmol Vis Sci. 2010;51(12):6496-6503.

48. Mao W, Tovar-Vidales T, Yorio T, Wordinger RJ, Clark AF. Perfusion-cultured bovine anterior segments as an ex vivo model for studying glucocorticoid-induced ocular hypertension and glaucoma. Invest Ophthalmol Vis Sci. 2011;52(11):8068-8075.

49. Zhang XY, Clark AF, Yorio T. Regulation of glucocorticoid responsiveness in glaucomatous trabecular meshwork cells by glucocorticoid receptor- $\beta$. Invest Ophthalmol Vis Sci. 2005;46(12):4607-4616.

50. Kumar S, Shah S, Deutsch ER, Tang HM, Danias J. Triamcinolone acetonide decreases outflow facility in C57BL/ 6 mouse eyes. Invest Ophthalmol Vis Sci. 2013;54(2):1280-1287.

51. Marciniak SJ, et al. CHOP induces death by promoting protein synthesis and oxidation in the stressed endoplasmic reticulum. Genes Dev. 2004; 18(24):3066-3077.

52. Gould DB, et al. Genetically increasing Myoc expression supports a necessary pathologic role of abnormal proteins in glaucoma. Mol Cell Biol. 2004; 24(20):9019-9025.

53. Shepard AR, et al. Glaucoma-causing myocilin mutants require the peroxisomal targeting signal-1 receptor (PTS1R) to elevate intraocular pressure. Hum Mol Genet. 2007;16(6):609-617.

54. Yam GH, Gaplovska-Kysela K, Zuber C, Roth J. Sodium 4-phenylbutyrate acts as a chemical chaperone on misfolded myocilin to rescue cells from endoplasmic reticulum stress and apoptosis. Invest Ophthalmol Vis Sci. 2007;48(4):1683-1690.

55 . He L, et al. ATM blocks tunicamycin-induced endoplasmic reticulum stress. FEBS Lett. 2009; 583(5):903-908. 